\title{
Effects of Remote Ischemic Conditioning Methods on Ischemia-Reperfusion Injury in Muscle Flaps: An Experimental Study in Rats
}

\author{
Durdane Keskin ${ }^{1}$, Ramazan Erkin Unlu ${ }^{2}$, Erkan Orhan ${ }^{3}$, Gamze Erkilinç ${ }^{4}$, Nihal Bogdaycioglu ${ }^{5}$, \\ Fatma Meric Yilmaz \\ ${ }^{1}$ Department of Plastic, Reconstructive and Aesthetic Surgery, Yuksek Ihtisas University, Ankara; ${ }^{2}$ Department of Plastic, Reconstructive and \\ Aesthetic Surgery, Ankara Numune Training and Research Hospital, Ankara; ${ }^{3}$ Department of Plastic Reconstructive and Aesthetic Surgery, \\ Namik Kemal University, Tekirdag; Departments of ${ }^{4}$ Pathology and ${ }^{5}$ Biochemistry, Ankara Numune Training and Research Hospital, \\ Ankara, Turkey
}

Background The aim of this study was to investigate the effects of remote ischemic conditioning on ischemia-reperfusion injury in rat muscle flaps histopathologically and biochemically.

Methods Thirty albino rats were divided into 5 groups. No procedure was performed in the rats in group 1, and only blood samples were taken. A gracilis muscle flap was elevated in all the other groups. Microclamps were applied to the vascular pedicle for 4 hours in order to achieve tissue ischemia. In group 2, no additional procedure was performed. In groups 3, 4, and 5 , the right hind limb was used and 3 cycles of ischemia-reperfusion for 5 minutes each (total, 30 minutes) was applied with a latex tourniquet (remote ischemic conditioning). In group 3, this procedure was performed before flap elevation (remote ischemic preconditoning). In group 4, the procedure was performed 4 hours after flap ischemia (remote ischemic postconditioning). In group 5, the procedure was performed after the flap was elevated, during the muscle flap ischemia episode (remote ischemic perconditioning).

Results The histopathological damage score in all remote conditioning ischemia groups was lower than in the ischemic-reperfusion group. The lowest histopathological damage score was observed in group 5 (remote ischemic perconditioning).

Conclusions The nitric oxide levels were higher in the blood samples obtained from the remote ischemic perconditioning group. This study showed the effectiveness of remote ischemic conditioning procedures and compared their usefulness for preventing ischemiareperfusion injury in muscle flaps.

\section{Keywords Ischemic preconditioning / Reperfusion injury / Ischemia}

Received: 4 Mar $2017 \bullet$ Revised: 5 Jul $2017 \bullet$ Accepted: 29 Aug 2017

pISSN: 2234-6163 • elSSN: 2234-6171 • https://doi.org/10.5999/aps.2017.44.5.384• Arch Plast Surg 2017;44:384-389
Correspondence: Durdane Keskin Department of Plastic Reconstructive and Aesthetic Surgery, Yuksek Intisas University, Muhsin Yazizicioglu Str. No: 10/25 Cukurambar, Cankaya, Ankara, Turkey Tel: $+90-5052889491$ Fax: +90-3325823046 E-mail: durdaneergun@gmail.com

No potential conflict of interest relevant to this article was reported.

\section{INTRODUCTION}

Flap loss continues to be an important problem in reconstruc- tive surgery $[1,2]$. The main causes are postoperative ischemia, hypoxia, and reperfusion following ischemia [3]. Ischemia/reperfusion $(\mathrm{I} / \mathrm{R})$ causes more tissue damage than is caused by 
ischemia [4]. Many studies have aimed to prevent I/R injuries [5-8]. Ischemic preconditioning (IPreC) is one of the most recent methods. The aim of IPreC is to increase the tolerance of the tissue to ischemia by performing brief controlled episodes of ischemia in the flap pedicle [9]. The effects of IPreC have been shown to be not only regional but also systemic. It is believed that the tolerance to ischemia in the target tissue can be increased through the remote ischemic conditioning (RIC) procedure due to the systemic effects of IPreC. Cyclic ischemia causes certain biochemical mediators to increase in the blood. One of these mediators is nitric oxide (NO) [10]. Its protective ability against I/R injury is thought to occur through the peripheral vasodilation induced by elevated NO levels. The effects of RIC have been shown in experiments conducted primarily in the myocardium, brain, liver, kidney, and skeletal muscle.

Despite the studies of the use of IPreC in flap surgery, the number of experimental studies on RIC is inadequate. Several experimental studies have shown the effectiveness of IPreC in flap surgery. However, direct IPreC produces trauma to major vessels and direct stress to the target organ. Because of this, the clinical use of IPreC is limited.

In this study, we investigated the feasibility of various RIC procedures in muscle flap surgery in light of the current literature. Their effects on histopathological and biochemical values were investigated experimentally, and the various methods were compared.

\section{METHODS}

\section{Animal preparation and care protocol}

The animal care and experimental procedures complied with the Principles of Laboratory Animal Care (Guide for the Care and Use of Laboratory Animals, National Institutes of Health Publication no. 86-23, revised 1996) guidelines and were approved by the institutional animal care and use committee of our university. Winstar-albino rats (240 to 300 g; Ankara University Animal Laboratory) were initially anesthetized by intramuscular ketamine $(40 \mathrm{mg} / \mathrm{kg})$ and xylazine $(10 \mathrm{mg} / \mathrm{kg})$. During the preischemic, ischemic, and initial 4 hours of reperfusion periods, animals were placed on a heated blanket with an overhead heat lamp to maintain body temperature at $37^{\circ} \mathrm{C}$. For the 48-hour reperfusion experiments, rats were returned to their cages in the vivarium and allowed access to water and chow ad libitum. Rats were kept on a 12-hour light-dark cycle, and the room temperature was kept constant between $21^{\circ} \mathrm{C}$ and $23^{\circ} \mathrm{C}$. After all measurements were completed, the rats were euthanized with an overdose of pentobarbital.

\section{Isolation of the gracilis muscle on its vascular pedicle}

The gracilis muscle and the neurovascular pedicle were dissected as described previously [11]. In brief, anesthesia was induced and the left groin was shaved. A $\times 3$ loupe was used for the dissection. An incision was made from the anterior iliac spine to the tip of the medial knee, exposing the femoral artery and the gracilis muscle. The gracilis muscle flap was elevated on its vascular pedicle using standard microsurgical techniques. This procedure isolated the gracilis pedicle and allowed us to induce complete ischemia in the muscle flap by clamping the vascular pedicle.

\section{Induction of ischemia in the muscle flap}

After dissection, a microvascular clamp was applied to the vascular pedicle below the origin of the gracilis pedicle for 4 hours (Fig. 1).

\section{Induction of remote ischemic condition}

Three cycles of 5 minutes of ischemia and 5 minutes of reperfusion were performed in the right hind limb by using a latex tourniquet (Fig. 2).

\section{Experimental groups}

Thirty rats were randomly assigned to 5 groups ( 6 animals per group). In group 1 , no surgery was performed, and only blood samples were taken. In group 2 (control), the vessels were clamped to induce 4 hours of ischemia and then opened for 48 hours of reperfusion. In group 3 (remote ischemic preconditioning, RIPreC), 3 cycles of 5-minute I/ $\mathrm{R}$ were performed before 4 hours of ischemia. In group 4 (remote ischemic postconditioning, RIPostC), 3 cycles of 5-minute I/R were performed after 4 hours of ischemia. In group 5 (remote ischemic perconditioning, RIPerC), 3 cycles of 5-minute I/R were performed after flap elevation, during 4 hours of ischemia (Table 1 ).

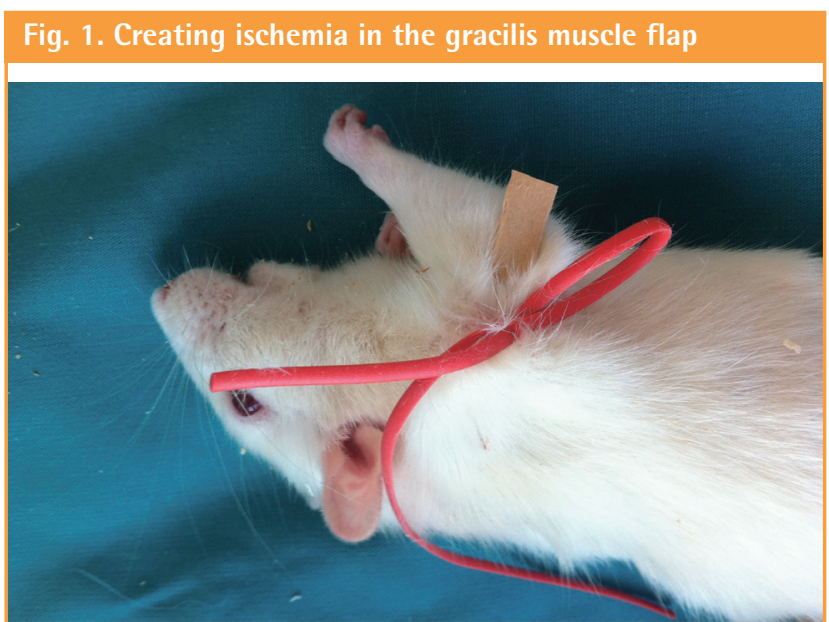




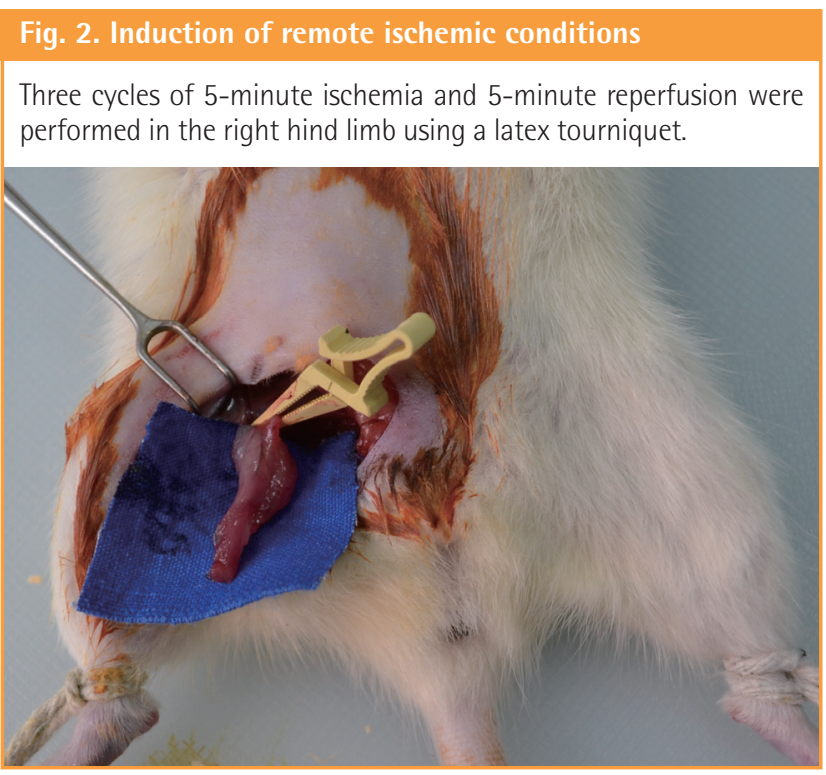

\section{Process}

The animals were allowed to awaken following reperfusion. They were allowed free access to chow and water and were monitored for 48 hours. After end of the 48 hours, intracardiac blood samples were taken for biomarker testing and the animals were euthanized. The entire gracilis muscle was resected with the neurovascular pedicle for histopathological investigation.

\section{Histopathological investigation}

The specimens were then placed in formaldehyde for 24 hours. The specimens were processed on an automatic tissue processor, embedded in paraffin, and $4-\mu \mathrm{m}$ sections were prepared. Specimens were stained with standard hematoxylin and eosin and examined by pathologists with no knowledge of the treatment group in a randomly selected manner. The histopathological parameters evaluated were loss in transverse ridging, migration of nuclei to the center, separation of the muscle fibers, interfascicular and intercellular edema, and lymphocyte infiltration in serial sections. Each group of findings was given a score: no change, 0 ; minimal change, 1 ; mild change, 2 ; moderate change, 3 ; significant change, 4 . Degenerative findings were also evaluated in 5 randomly selected areas from each section in a computer environment using the Leica QWin (V3 plus image) image (Leica Microsystems, Wetzlar, Germany) analysis system. The histopathological degenerative scores of the groups were evaluated.

\section{Biochemical investigation}

Blood samples taken immediately before sacrifice were placed in dry tubes and centrifuged at 3,000 rpm for 10 minutes. Serum NO levels were measured with the enzyme-linked immu-

\section{Table 1. Distribution of the groups}

\begin{tabular}{ll}
\hline Group 1 & No procedure, only blood samples were taken \\
Group 2 (control) & $4 \mathrm{hr}$ of gracilis muscle flap ischemia \\
& $48 \mathrm{hr}$ of reperfusion \\
Group 3 (RIPreC) & 3 cycles of I/R for 5 min (total $30 \mathrm{~min}$ ) \\
& $4 \mathrm{hr}$ of gracilis muscle flap ischemia \\
& $48 \mathrm{hr}$ of reperfusion \\
Group 4 (RIPostC) & $4 \mathrm{hr}$ of gracilis muscle flap ischemia \\
& $3 \mathrm{cycles}$ of I/R for 5 min (total $30 \mathrm{~min}$ ) \\
& $48 \mathrm{hr}$ of reperfusion \\
Group 5 (RIPerC) & $\begin{array}{l}\text { During } 4 \text { hours of gracilis muscle flap ischemia, } \\
3 \mathrm{cycles} \text { of I/R for } 5 \text { min (for the first } 30 \text { min of muscle flap } \\
\text { ischemia) }\end{array}$ \\
& $48 \mathrm{hr}$ of reperfusion
\end{tabular}

$\mathrm{RIPreC}$, remote ischemic preconditioning; I/R, ischemia/reperfusion; RIPostC, remote ischemic postconditioning; RIPerC, remote ischemic perconditioning.

nosorbent assay (ELISA) method. The analysis was performed using the EASTBIOPHARM (Hangzhou, China) nitric oxide synthase inducible (INOS-NOS2) ELISA kit (catalog number: CK-E90762) (measurement range, 02-60 ng/mL).

\section{Statistical evaluation}

Descriptive statistics for the relevant characteristics were expressed as median, mean, standard deviation, and minimum and maximum values. The Kruskal-Wallis test was used to compare the groups in terms of these characteristics. Following the Kruskal-Wallis test, the Dunn test was performed to determine the groups responsible for the differences. The statistical significance level was accepted as $5 \%(\mathrm{P}<0.05)$.

\section{RESULTS}

\section{Biochemical evaluation results}

Serum NO levels were statistically significantly higher only in group 5 (RIPerC) compared to group 1 and group 2 (control) $(\mathrm{P}=0.012)$ (Table 2, Fig. 3).

\section{Histopathological investigation results}

The separation of the muscle fibers $(\mathrm{P}=0.001)$ and interfascicular and intercellular edema $(\mathrm{P}=0.012)$ parameters were lower in all RIC groups (groups 3, 4, and 5) than in the control group (group 2). The lymphocyte infiltration parameter was lower in group 3 (RIPreC) and group 5 (RIPerC) than in the control group. The loss in transverse ridging parameter was lower in group 4 (RIPostC) and group 5 (RIPerC). A significant decrease in the migration of the nuclei to the center was only observed in the RIPostC group compared to the control group $(\mathrm{P}=0.015)$ (Table 3$)$. The collective evaluation of histopathological parameters revealed a decrease in the ischemia reperfu- 
sion degenerative damage score in the muscle flaps in which the RIPerC, RIPreC, or RIPostC method was used (Fig. 4).

\section{DISCUSSION}

Injuries caused by reperfusion after prolonged ischemia are a significant problem in reconstructive surgery (3). Many studies have aimed to prevent $\mathrm{I} / \mathrm{R}$ injury. Several drugs and procedures have been tried in order to prevent I/R injury, and IPreC is one of the important current methods.

IPreC, in which brief episodes of non-damaging ischemia in-

\section{Table 2. Descriptive statistics of nitric oxide levels in the} groups

\begin{tabular}{|c|c|c|c|c|c|}
\hline Group & Median & Mean \pm SD & Min. & Max. & $\mathrm{P}$ \\
\hline Group 1 & $84.65^{a)}$ & $216.68 \pm 245.51$ & 50.08 & 665.43 & 0.012 \\
\hline Group 2 (control) & $96.89^{a)}$ & $147.61 \pm 126.78$ & 29.78 & 379.00 & \\
\hline Group 3 (RIPreC) & $119.49^{\mathrm{a})}$ & $316.37 \pm 375.4$ & 73.28 & 1014.28 & \\
\hline Group 4 (RIPostC) & $64.64^{\text {a) }}$ & $78.87 \pm 39.23$ & 43.55 & 147.81 & \\
\hline Group 5 (RIPerC) & $1,443.52^{b)}$ & $1,510.2 \pm 1,312.72$ & 191.74 & $3,760.38$ & \\
\hline
\end{tabular}

SD, standard deviation; Min, Minimum; Max, Maximum; RIPreC, remote ischemic preconditioning; RIPostC, remote ischemic postconditioning; RIPerC, remote ischemic percondirioning.

${ }^{a, b}$ Different letters represent statistically significant differences among the median values of the groups $(P<0.05)$. duce protection against a subsequent, prolonged, lethal ischemic period, can be defined as an endogenous mechanism that protects against I/R injury. Previous IPreC studies in rat models have shown that enhancement of flap survival and improvement

\section{Fig. 3. Graphical demonstration of the NO values between groups.}

Graphical demonstration of the nitric oxide (NO) values between groups. RIPreC, remote ischemic preconditioning; RIPostC, remote ischemic postconditioning; RIPerC, remote ischemic perconditionind. *Difference from other groups is statistically significant.

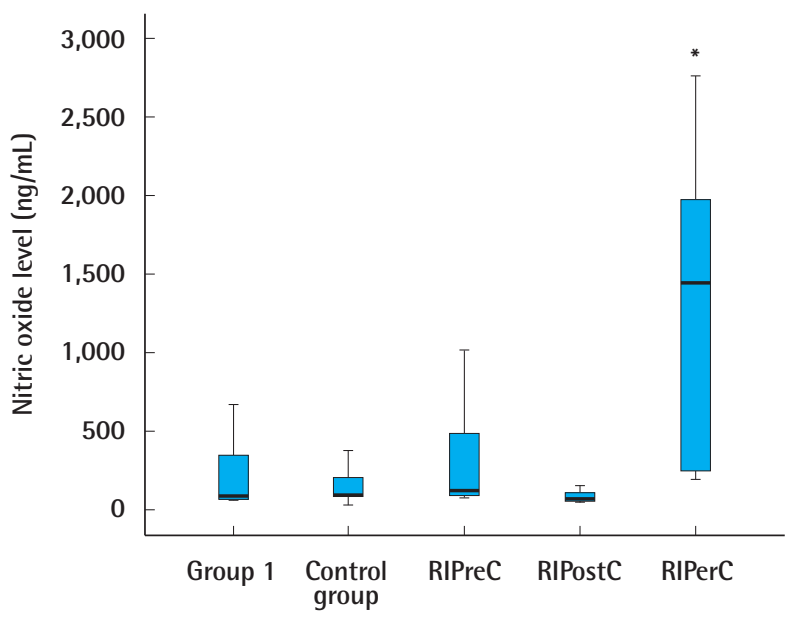

Table 3. Comparison of the histopathological degenerative scores of the groups

\begin{tabular}{|c|c|c|c|c|c|c|c|c|c|}
\hline \multirow{2}{*}{ Histopathological parameters } & \multicolumn{2}{|c|}{ Group 2 (control) } & \multicolumn{2}{|c|}{ Group 3 (RIPreC) } & \multicolumn{2}{|c|}{ Group 4 (RIPostC) } & \multicolumn{2}{|c|}{ Group 5 (RIPerC) } & \multirow{2}{*}{ P-value } \\
\hline & Mean & Median & Mean & Median & Mean & Median & Mean & Median & \\
\hline Loss in transverse ridging & $3.00 \pm 0.63$ & $3.0^{\text {a) }}$ & $2.50 \pm 0.55$ & $2.5^{\mathrm{a}, \mathrm{b})}$ & $1.55 \pm 0.55$ & $1.5^{c)}$ & $2.00 \pm 0$ & $2.0^{b, c)}$ & $0.003^{*}$ \\
\hline Migration of nuclei to the center & $0.83 \pm 0.41$ & $1.0^{\mathrm{a})}$ & $1.00 \pm 0$ & $1.0^{\mathrm{a})}$ & $0.33 \pm 0.52$ & $0^{\text {b) }}$ & $1.00 \pm 0$ & $1.0^{\mathrm{a})}$ & $0.015^{*}$ \\
\hline Separation of the muscle fibers & $3.17 \pm 0.41$ & $3.0^{\text {a) }}$ & $2.00 \pm 0$ & $2.0^{\mathrm{b})}$ & $1.67 \pm 0.52$ & $2.0^{b)}$ & $1.67 \pm 0.52$ & $2.0^{b)}$ & $0.001^{*}$ \\
\hline İnterfascicular and intercellular edema & $3.00 \pm 0.63$ & $2.0^{\text {a) }}$ & $1.83 \pm 0.41$ & $2.0^{\mathrm{b})}$ & $2.33 \pm 0.52$ & $2.0^{\mathrm{b})}$ & $2.17 \pm 0.41$ & $2.0^{\mathrm{b})}$ & $0.012^{\star}$ \\
\hline Lymphocyte infiltration & $3.17 \pm 0.98$ & $3.5^{\text {a) }}$ & $2.67 \pm 0.82$ & $2.5^{\mathrm{b}, \mathrm{c})}$ & $2.83 \pm 0.75$ & $3.0^{\mathrm{a}, \mathrm{b})}$ & $2.00 \pm 0.64$ & $2.0^{\text {c) }}$ & $0.047^{*}$ \\
\hline
\end{tabular}

RIPreC, remote ischemic preconditioning; RIPostC, remote ischemic postconditioning; RIPerC, remote ischemic percondirioning.

a,b,cid Different letters represent statistically different amoung the groups in the same row. ${ }^{*}<<0.05$.

Fig. 4. Microscopic muscle morphology of the groups $\left(H \& E_{1} \times 200\right)$

(A) Intercellular edema and lymphocyte infiltration in the control group. (B) Intercellular edema and lymphocyte infiltration in group 3 (RIPreC). (C) Intercellular edema and lymphocyte infiltration in group 4 (RIPostC). (D) Intercellular edema and lymphocyte infiltration in group 5 (RIPerC). $\mathrm{RIPreC}$, remote ischemic preconditioning; RIPostC, remote ischemic postconditioning; RIPerC, remote ischemic perconditioning; red arrows, lymphocyte infiltration.
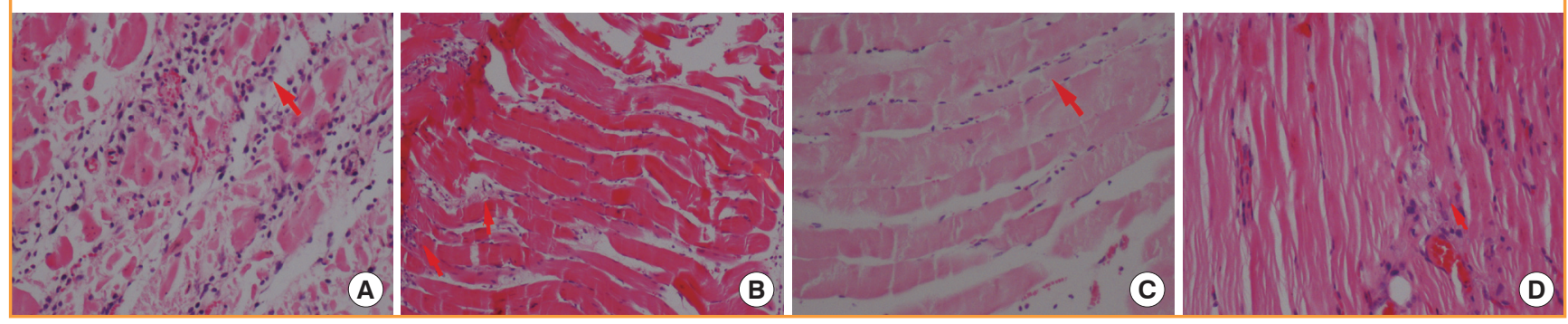
of flap microcirculation following ischemia can be achieved not only by preclamping the flap pedicle but also by inducing a controlled I/R event in a body area distant from the flap (limb ischemia) prior to its preparation $[12,13]$.

Although the effectiveness of IPreC has been shown in experimental studies, its use has been limited due to the possibility of damage caused by clamping the pedicle of the tissue to be transferred and the tissue being exposed to ischemia. Obtaining the same effectiveness with RIC, a non-invasive method, makes clinical use reasonable.

The muscle flap protective effect of the non-invasive RIPreC procedure against I/R injury was shown by Addison et al. [14] in experimental studies. The efficiency of RIPostC, which can specifically be used in ischemia that develops as a result of trauma or stroke where preconditioning is not possible, in clinical use was first experimentally shown by Andreka et al. [15] in pig myocardial muscle. Performing the procedure after ischemia is thought to decrease reperfusion injury. The protective effect of this procedure against I/ $\mathrm{R}$ damage was demonstrated by Schmidt et al. [16] in their study, in which they created non-invasive intermittent ischemia (RIPerC) during myocardial infarction. The effect of RIPreC in flap surgery has been shown previously $[13,17,18]$. However, no studies have been conducted to assess the effects of RIPerC and RIPostC on muscle flaps, and these 3 methods have not been compared.

We experimentally evaluated the effectiveness of the RIPreC, RIPostC, and RIPerC methods and compared them to each other in terms of degenerative histopathological findings and biochemical parameters in this study. We concluded that all 3 methods clearly provided protection against I/R injury.

Histopathologically, nuclear internalization, hyalinization with loss in transverse ridging, degeneration of fibers, edema, and lymphocyte infiltration are seen during an I/R injury [19]. One of the most common pathological abnormalities observed in muscle biopsies is nuclear internalization, which seems to be a reaction to virtually any type of injury. In serial sections of hyaline fibers, areas of necrosis may be found, indicating the importance of loss of transverse ridging and separation of muscle fibers as a sign of fiber destruction [20].

Evaluation of the mean histopathological scores revealed that all 3 methods were protective against I/R injury compared to group 2 (control), but the best flap histopathologic score was observed for the RIPerC method.

Among the other two methods, RIPreC had a better histopathologic score than RIPostC. Based on the demonstrated effects, these techniques may be useful during flap surgical procedures, especially muscle flap surgery. Although RIPostC was the least effective of the 3 methods, it can be used during replanta- tion surgery or surgery on a flap exposed to ischemia, and may help in increasing flap viability.

The systemic effect of RIC has not been fully clarified. Studies have shown that endogenous NO plays a role in the RIC mechanism $[21,22]$. NO has been reported to play a central role in the mechanism of ischemic preconditioning. Peralta et al. [23] demonstrated in a model of hepatic I/R injury that adenosine or NO administration prior to ischemia simulated the effect of preconditioning, whereas inhibition of NO synthesis eliminated the protective effect of hepatic preconditioning. The findings of Peralta et al. gave us cause to determine further the role of NO in the mechanisms of both 'classic' and acute RIPreC of flaps.

In contrast, the protective effect of ischemic preconditioning was observed to be blocked in studies where endogenic NO synthesis was inhibited. Wang et al. [24] demonstrated in a cremaster flap rat model that the application of sodium nitroprusside (a NO donor) had the same preconditioning effect as ischemic preconditioning via preclamping, whereas the effect was eliminated when NOS was blocked by L-NAME.

We found the blood NO level in the RIPerC group to be the highest among all groups, including group 1 and 2 . RIPreC was found to increase blood NO, but no significant increase was observed in the RIPostC group. These findings highlight that ischemia in 2 locations simultaneously can lead to significant increases in serum NO levels.

The effect on flap viability was correlated with the histopathological results, which is thought to possibly be related to the increased NO synthesis in the blood.

Since RIC is a non-invasive method, it does not harm the patient or the flap. Its use has no additional cost. It exerts its effect without any drug administration to the body or the tissue, unlike other conditioning methods that have previously been used experimentally but for which clinical use has not been common. Therefore, problems such as dose limitation are not encountered during the use of this technique.

The remote ischemic conditioning methods that can be used to reduce I/R injury, especially in muscle flap surgery, are inexpensive, non-invasive, and reliable methods for improving flap survival.

\section{ORCID}

Durdane Keskin https://orcid.org/0000-0002-4011-1931

\section{REFERENCES}

1. Lorenzo AR, $\mathrm{Lin} \mathrm{CH}$, Lin $\mathrm{CH}$, et al. Selection of the recipient vein in microvascular flap reconstruction of the lower 
extremity: analysis of 362 free-tissue transfers. J Plast Reconstr Aesthet Surg 2011;64:649-55.

2. Nahabedian MY, Momen B, Manson PN. Factors associated with anastomotic failure after microvascular reconstruction of the breast. Plast Reconstr Surg 2004;114:74-82.

3. Moran SL, Serletti JM. Outcome comparison between free and pedicled TRAM flap breast reconstruction in the obese patient. Plast Reconstr Surg 2001;108:1954-60.

4. Loerakker S, Oomens CW, Manders E, et al. Ischemia-reperfusion injury in rat skeletal muscle assessed with T2weighted and dynamic contrast-enhanced MRI. Magn Reson Med 2011;66:528-37.

5. Dillon JP, Laing AJ, Cahill RA, et al. Activated protein C attenuates acute ischaemia reperfusion injury in skeletal muscle. J Orthop Res 2005;23:1454-9.

6. Zheng J, Wang R, Zambraski E, et al. Protective roles of adenosine $\mathrm{A} 1, \mathrm{~A} 2 \mathrm{~A}$, and $\mathrm{A} 3$ receptors in skeletal muscle ischemia and reperfusion injury. Am J Physiol Heart Circ Physiol 2007;293:H3685-91.

7. Arato E, Kurthy M, Sinay L, et al. Effect of vitamin E on reperfusion injuries during reconstructive vascular operations on lower limbs. Clin Hemorheol Microcirc 2010;44:125-36.

8. Tatlidede SH, Murphy AD, McCormack MC, et al. Improved survival of murine island skin flaps by prevention of reperfusion injury. Plast Reconstr Surg 2009;123:1431-9.

9. Murry CE, Jennings RB, Reimer KA. Preconditioning with ischemia: a delay of lethal cell injury in ischemic myocardium. Circulation 1986;74:1124-36.

10. Koti RS, Seifalian AM, McBride AG, et al. The relationship of hepatic tissue oxygenation with nitric oxide metabolism in ischemic preconditioning of the liver. Faseb j 2002;16: 1654-6.

11. Dautel G, Braga da Silva J, Merle M. Pedicled or free flap transfer of the gracilis muscle in rats. J Reconstr Microsurg 1991;7:23-5.

12. Kuntscher MV, Schirmbeck EU, Menke H, et al. Ischemic preconditioning by brief extremity ischemia before flap ischemia in a rat model. Plast Reconstr Surg 2002;109: 2398-404.

13. Kuntscher MV, Kastell T, Sauerbier M, et al. Acute remote ischemic preconditioning on a rat cremasteric muscle flap model. Microsurgery 2002;22:221-6.

14. Addison PD, Neligan PC, Ashrafpour H, et al. Noninvasive remote ischemic preconditioning for global protection of skeletal muscle against infarction. Am J Physiol Heart Circ Physiol 2003;285:H1435-43.

15. Andreka G, Vertesaljai M, Szantho G, et al. Remote ischaemic postconditioning protects the heart during acute myocardial infarction in pigs. Heart 2007;93:749-52.

16. Schmidt MR, Smerup M, Konstantinov IE, et al. Intermittent peripheral tissue ischemia during coronary ischemia reduces myocardial infarction through a KATP-dependent mechanism: first demonstration of remote ischemic perconditioning. Am J Physiol Heart Circ Physiol 2007;292: H1883-90.

17. Kuntscher MV, Hartmann B, Germann G. Remote ischemic preconditioning of flaps: a review. Microsurgery 2005;25: 346-52.

18. Kuntscher MV, Kastell T, Engel H, et al. Late remote ischemic preconditioning in rat muscle and adipocutaneous flap models. Ann Plast Surg 2003;51:84-90.

19. Labbe R, Lindsay T, Walker PM. The extent and distribution of skeletal muscle necrosis after graded periods of complete ischemia. J Vasc Surg 1987;6:152-7.

20. Mills SE. Histology for pathologists. 3rd ed. Philadelphia: Lippincott Williams \& Wilkins; 2007.

21. Küntscher MV, Juran S, Altmann J, et al. Role of nitric oxide in the mechanism of preclamping and remote ischemic preconditioning of adipocutaneous flaps in a rat model. J Reconstr Microsurg 2003;19:55-60.

22. Kuntscher MV, Kastell T, Altmann J, et al. Acute remote ischemic preconditioning II: the role of nitric oxide. Microsurgery 2002;22:227-31.

23. Peralta C, Closa D, Hotter G, et al. Liver ischemic preconditioning is mediated by the inhibitory action of nitric oxide on endothelin. Biochem Biophys Res Commun 1996;229: 264-70.

24. Wang WZ, Anderson GL, Guo SZ, et al. Initiation of microvascular protection by nitric oxide in late preconditioning. $\mathrm{J}$ Reconstr Microsurg 2000;16:621-8. 